\title{
NON-LINEAR ELASTIC THERMAL STRESS ANALYSIS WITH PHASE CHANGES
}

\author{
S. AMADA and W.H. YANG \\ Department of Applied Mechanics and Engineering Science, The University of Michigan, Ann Arbor, Michigan 48109, USA
}

Received 6 January 1978

\begin{abstract}
The non-linear elastic, thermal stress analy sis with temperature induced phase changes in the materials is presented. An infinite plate (or body) with a circular hole (or tunnel) is subjected to a thermal loading on its inner surface. The peak temperature around the hole reaches beyond the melting point of the material. The non-linear diffusion equation is solved numerically using the finite difference method. The material properties change rapidly at temperatures where the change of crystal structures and solid-liquid transition occur. The elastic stresses induced by the transient non-homogeneous temperature distribution are calculated. The stresses change remarkably when the phase changes occur and there are residual stresses remaining in the plate after one cycle of thermal loading.
\end{abstract}

\section{Introduction}

The interest in thermal stresses with phase changes has been promoted from the demands of welding industries [1] and from the development of fast breeder nuclear reactors [2].

Two types of phase changes induced by temperature change are common in crystlline materials: the changes of crystal structures (face-centered cubic to bodycentered cubic and vice versa) and the solid-liquid transition. The phenomena of changes of crystal structures and associated changes of mechanical properties are utilized in the heat treatment of metals such as quenching and annealing. The solid-liquid transition is essential to the process of welding [3] and an important consideration in the safety and accident analysis [4] of nuclear reactors, so-called 'melt down analysis'.

It is well known that the non-uniform temperature distribution introduces stresses in solids and that phase transformations add complexity to thermal stress distributions. Assuming that the coupling effect can be neglected, it is possible to calculate the temperature and stress fields sequentially rather than simultaneously.

Investigations on such a model problem can answer most of the questions on heat conduction and stress analysis associated with phase changes.

In the literature on heat conduction there exist many works concerning phase changes. The melting problem modeled as the Stefan problem can be traced back to much earlier studies of such phenomena, as indicated by Carslaw and Jaeger [5].

The area that involves problems associated with phase changes spurs active research, and recent studies are listed in the survey by Boley [6]. The monograph edited by Ockendon and Hodgkins [7] treated this subject as a moving boundary value problem in view of a numerical scheme.

Comparatively few studies have been reported on the subject of stress analysis under phase changes. A few such studies are referenced in the review [8] by Perzyna and Sawczuk.

Phase changes of materials introduce conspicuous characteristics on thermal and mechanical properties which appear as variable coefficients in the differential equations. Those coefficients are functions of temperature. Some of them show jumps or spike-like discontinuous behavior around the phase changes.

To summarize the work done on this subject, the literature may be divided into three categories. The first considered only the change of crystal structures of the materials. Based on a simple assumption such structural change is interpreted as the local change of volume [9-12], temperature-dependency of the Young's modulus [13], and the change of thermal expansion coefficient [14]. The stress analysis with 
the solid-liquid phase changes belongs to the second category. The work done in this category consists of the melting problem with temperature-dependent yield stress [15], and of the solidification problems with constant properties $[16,17]$, with variable Poisson's ratio [18], with variable Young's modulus [19] and with the temperature-dependency of yield stress [20]. The third category of studies treated both the change of crystal structure and liquid-solid phase changes. The effects of those phase changes on the temperature field as well as the stress field were considered in $[21,22]$.

The present study belongs to the third category. It concerns the stress analysis of an infinite plate with a circular hole, the boundary of which is heated above the melting temperature of the material and then cooled down to room temperature. The temperaturedependent thermal and mechanical properties, including two types of phase changes, are used to calculate the temperature distribution and elastic stresses in the plate. The similar plane strain problem can be solved in the same way.

\section{Analysis}

For the transient heating and cooling cycle, the high temperature region is confined to a region near the hole. Let the plate be divided into two regions (I and II) separated by a circle of radius $r=b$ as shown

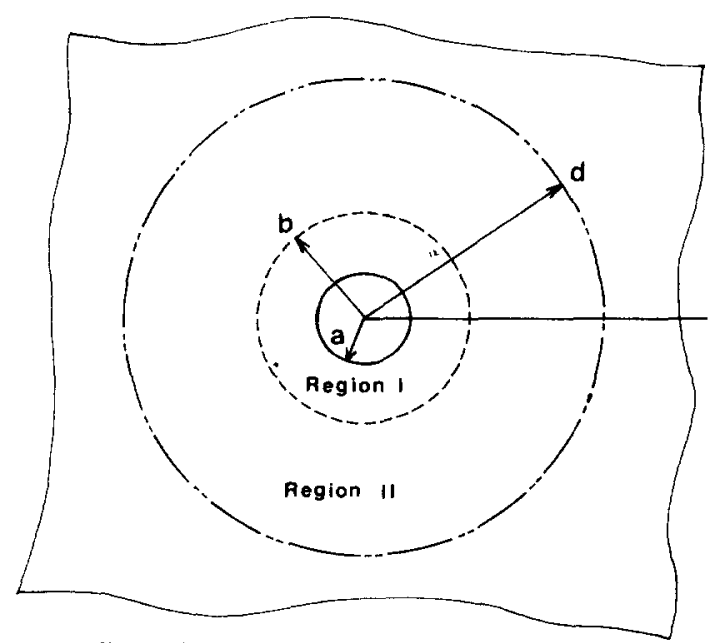

Fig. 1. Geometry and notations of a plate. in fig. 1 where the radius $b$ is chosen so that the temperature in region II remains moderate at all tine and the radius $d$ is chosen large enough so that the temperature at this radius can be assumed to remain at a constant ambient temperature and that the radius stress can be assumed zero. In region II, $b \leqslant r \leqslant d$, the influence of the temperature on all mechanical and thermal properties can be neglected, therefore the solutions in this region are governed by linear differential equations. In region $\mathrm{I}, a \leqslant r \leqslant b$, where the temperature is high enough to change the material properties, the differential equations are non-linear.

\subsection{Temperature field}

For a transient heat flow in a medium whose conductivity $K$, density $\rho$ and specific heat $C$ are temperature dependent, the equation of heat conduction in region $I$ is

$\operatorname{div}[K(T) \operatorname{grad} T]=\rho(T) C(T) \frac{\partial T}{\partial t}$,

with the initial temperature

$T=T_{0}=$ constant,$\quad$ at $t=0$,

and with the boundary condition

$-K(T) \frac{\partial T}{\partial r}=h\left(T-T_{\mathrm{s}}\right) \quad$ at $r=a$,

where $h$ is a heat transfer coefficient and $T_{\mathbf{s}}$ is the temperature of the medium inside the hole.

Since all the mechanical and thermal properties are assumed to be independent of temperature in region II, we can apply the linear differential equation

$K_{0} \Delta T^{\mathrm{II}}=\rho_{0} C_{0} \frac{\partial T^{\mathrm{II}}}{\partial t}$,

where $K_{0}, \rho_{0}$ and $C_{0}$ are the values of $K, \rho$ and $C$ at $T=T_{0}$, and $\Delta$ is the Laplace operator. The initial and boundary conditions are

$T^{\mathrm{II}}=T_{0}, \quad$ at $t=0$,

$T^{\mathrm{II}}=T_{0}, \quad$ at $r=b$.

The continuity of the temperature and heat flow at the interface between the two regions require the following two conditions:

$T=T^{\mathrm{II}}, \quad$ at $r=b$, 
$K \frac{\partial T}{\partial r}=K_{0} \frac{\partial T^{\mathrm{Il}}}{\partial r}, \quad$ at $r=b$ lows:

We introduce the dimensionless quantities as fol-

$\bar{r}=\frac{r}{b} ; \quad \bar{a}=\frac{a}{b} ; \quad \bar{d}=\frac{d}{b}$,

$\bar{T}=\frac{T}{T_{0}} ; \quad \bar{T}_{0}=1 ; \quad \bar{T}_{\mathrm{s}}=\frac{T_{\mathrm{s}}}{T_{0}}$,

$\bar{K}=\frac{K}{K_{0}} ; \quad \bar{\rho}=\frac{\rho}{\rho_{0}} ; \quad \bar{C}=\frac{C}{C_{0}}$,

$\mathrm{Bi}=\frac{h b}{K_{0}} ; \quad \bar{t}=\frac{K_{0} t}{b^{2} \rho_{0} C_{0}}$,

where $\mathrm{Bi}$ and $t$ are non-dimensional parameters called the Biot number and the Fourier number. The equations and conditions are rewritten in dimensionless form:

for region I:

$\operatorname{div}[\bar{K}(\bar{T}) \operatorname{grad} \bar{T}]=\bar{\rho}(\bar{T}) \bar{C}(\bar{T}) \frac{\partial \bar{T}}{\partial \bar{t}}$,

$\bar{T}=1, \quad$ at $\bar{t}=0$,

$-\bar{K}(\bar{T}) \frac{\partial \bar{T}}{\partial \bar{r}}=\operatorname{Bi}\left(\bar{T}-\bar{T}_{\mathrm{s}}\right), \quad$ at $\bar{r}=\bar{a} ;$

for region II:

$\Delta \bar{T}^{\mathrm{II}}=\frac{\partial \bar{T}^{\mathrm{II}}}{\partial \bar{t}}$,

$\bar{T}^{\mathrm{II}}=1, \quad$ at $\bar{t}=0$,

$\bar{T}^{\mathrm{II}}=1, \quad$ at $\bar{r}=d$.

The continuity conditions become

$\bar{T}=\bar{T}^{\mathrm{II}}, \quad$ at $\bar{r}=1$,

$\bar{K} \frac{\partial \bar{T}}{\partial \bar{r}}=\frac{\partial \bar{T}^{\mathrm{Il}}}{\partial \bar{r}}, \quad$ at $\bar{r}=1$.

Assuming that $\bar{T}(\bar{r}=1, \bar{t})$ is known, the solution in region II can be obtained by the Duhamel's theorem
[23]:

$$
\begin{aligned}
& \bar{T}^{\mathrm{ll}}(\bar{r}, \bar{t})=1-\pi \sum_{n=0}^{\infty} \lambda_{n}^{2} B_{n} U_{0}\left(\lambda_{n} \bar{r}\right) \\
& \quad \times \exp \left(-\lambda_{n}^{2} \bar{t}\right) \int_{0}^{\bar{t}} \bar{T}(\bar{r}=1, \tau) \exp \left(\lambda_{n}^{2} \tau\right) \mathrm{d} \tau,
\end{aligned}
$$

where $\lambda_{n}$ is the $n$th root of $\lambda$ satisfying the equation

$J_{0}(\lambda) Y_{0}(\lambda \bar{d})-Y_{0}(\lambda) J_{0}(\lambda \bar{d})=0$,

$U_{0}\left(\lambda_{n} \bar{r}\right)=J_{0}\left(\lambda_{n} \bar{r}\right) Y_{0}\left(\lambda_{n} \bar{d}\right)-Y_{0}\left(\lambda_{n} \bar{r}\right) J_{0}\left(\lambda_{n} \bar{d}\right)$,

$B_{n}=\frac{J_{0}\left(\lambda_{n}\right) J_{0}\left(\lambda_{n} \bar{d}\right)}{J_{0}^{2}\left(\lambda_{n}\right)-J_{0}^{2}\left(\lambda_{n} \bar{d}\right)}$,

and $J_{0}$ and $Y_{0}$ are the Bessel and Neumann functions of zeroth order. The solution, eq. (13), satisfies automatically the first condition of eq. (12). The second condition of eq. (12) requires

$$
\begin{aligned}
& \bar{K} \frac{\partial \bar{T}}{\partial \bar{r}}=-\pi \sum_{n=0}^{\infty} \lambda_{n}^{3} B_{n} U_{1}\left(\lambda_{n} \bar{r}\right) \\
& \quad \times \exp \left(-\lambda_{n}^{2} \bar{t}\right) \int_{0}^{\bar{t}} \bar{T}(\bar{r}=1, \tau) \exp \left(\lambda_{n}^{2} \tau\right) \mathrm{d} \tau, \text { at } \bar{r}=1
\end{aligned}
$$

where $U_{1}$ is

$U_{1}\left(\lambda_{n} \bar{r}\right)=Y_{1}\left(\lambda_{n} \bar{r}\right) J_{0}\left(\lambda_{n} \bar{d}\right)-J_{1}\left(\lambda_{n} \bar{r}\right) Y_{0}\left(\lambda_{n} \bar{d}\right)$,

and $J_{1}$ and $Y_{1}$ are the Bessel and Neumann functions of the first order. With the condition eq. (15) added to eq. (10) we have the complete set of differential equations with the initial and boundary conditions for the solution in region I. It is noted that the boundary condition eq. (15) is history dependent.

The non-linear diffusion equation is transformed into the finite difference equation. Let the space and time be divided into the appropriate intervals. Then $i$ denotes the $i$ th discrete point on a radial axis and $j$ denotes the $j$ th discrete point in the time axis. Applying the central and backward difference formulae to the space derivative and the time derivative, respectively, in eq. (10),

$$
\begin{aligned}
\frac{\partial}{\partial \bar{r}}\left[\bar{K}(\bar{T}) \frac{\partial \bar{T}}{\partial \bar{r}}\right] \cong\left[\frac{\bar{K}(i+1, j)+\bar{K}(i, j)}{2} \frac{\bar{T}(i+1, j)-\bar{T}(i, j)}{\bar{r}(i+1)-\bar{r}(i)}\right. \\
\left.\quad-\frac{\bar{K}(i, j)+\bar{K}(i-1, j)}{2} \cdot \frac{\bar{T}(i, j)-\bar{T}(i-1, j)}{\bar{r}(i)-\bar{r}(i-1)}\right] /
\end{aligned}
$$




$$
\frac{\bar{r}(i+1)-\bar{r}(i-1)}{2}
$$

and

$$
\begin{aligned}
\bar{\rho}(\bar{T}) \bar{C}(\bar{T}) \frac{\partial \bar{T}}{\partial \bar{t}} \cong \frac{\bar{\rho}(i, j-1)+\bar{\rho}(i, j)}{2} \frac{\bar{C}(i, j-1)+\bar{C}(i, j)}{2} \\
\quad \times \frac{\bar{T}(i, j)-\bar{T}(i, j-1)}{\bar{t}(j)-\bar{t}(j-1)}
\end{aligned}
$$

are obtained. In matrix form the finite difference equations are given by

$$
[A]\{T\}=\{B\},
$$

where $[A]$ is the coefficient matrix which we can calculate from the geometry of the plate and from the thermal properties of the material; $\{T\}$ is the vector whose components are the unknown temperatures at the discrete points; and $\{B\}$ is the known vector which changes with the time step.

\subsection{Stress field}

It is assumed that the plane stress state holds and that the strains are small. We shall perform stress analysis in the same two regions used previously for thermal analysis.

For region I we have the equilibrium equation

$\frac{\partial \sigma_{r}}{\partial r}+\frac{\sigma_{r}-\sigma_{\theta}}{r}=0$

with the initial condition

$\sigma_{r}=\sigma_{\theta}=0, \quad$ at $t=0$

and the boundary condition

$\sigma_{r}=0, \quad$ at $r=a$.

Here $\sigma_{r}$ and $\sigma_{\theta}$ are the radial and circumferential stresses, respectively. In addition, we have the compatibility equation for the axisymmetric case

$\frac{\partial \epsilon_{\theta}}{\partial r}+\frac{\epsilon_{\theta}-\epsilon_{r}}{r}=0$,

where $\epsilon_{r}$ and $\epsilon_{\theta}$ denote the radial and circumferential strains, respectively. The stress-strain relation is given by

$\{\epsilon\}=[D]^{-1}\{\sigma\}+\left\{\epsilon^{\mathrm{T}}\right\}$, where

$$
\begin{array}{cc}
\{\epsilon\}=\left\{\begin{array}{c}
\epsilon_{r} \\
\epsilon_{\theta}
\end{array}\right\} ; \quad\{\sigma\}=\left\{\begin{array}{c}
\sigma_{r} \\
\sigma_{\theta}
\end{array}\right\} ; \quad\left\{\epsilon^{\mathrm{T}}\right\}=\left\{\begin{array}{ll}
\alpha(T) & T \\
\alpha(T) & T
\end{array}\right\}, \\
{[D]^{-1}=\frac{1}{E(T)}\left\{\begin{array}{rr}
1 & -\nu \\
-\nu & 1
\end{array}\right\},}
\end{array}
$$

$\alpha$ is the thermal expansion coefficient and $E$ is Young's modulus. It is assumed that the Poisson's ratio $v$ is constant in the solid phase.

For region II, we have the equilibrium equation

$\frac{\partial \sigma_{r}^{\mathrm{II}}}{\partial r}+\frac{\sigma_{r}^{\mathrm{II}}-\sigma_{\theta}^{\mathrm{II}}}{r}=0$

with the initial condition

$\sigma_{r}^{\mathrm{II}}=\sigma_{\theta}^{\mathrm{II}}=0, \quad$ at $t=0$,

and with the boundary condition

$\sigma_{r}^{\text {II }}=0, \quad$ at $r=d$.

The compatibility equation is

$\frac{\partial \epsilon_{\theta}^{\mathrm{II}}}{\partial r}+\frac{\epsilon_{\theta}^{\mathrm{II}}-\epsilon_{r}^{\mathrm{II}}}{r}=0$.

Under the assumption of constant material properties in region II, the stress-strain relation becomes

$\left\{\epsilon^{\mathrm{II}}\right\}=\left[D_{0}\right]^{-1}\left\{\sigma^{\mathbf{I I}}\right\}+\left\{\epsilon_{0}^{\mathbf{T}}\right\}$,

where

$$
\begin{array}{cc}
\{\epsilon\}=\left\{\begin{array}{c}
\epsilon_{r}^{\mathrm{II}} \\
\epsilon_{\theta}^{\mathrm{II}}
\end{array}\right) ; \quad\left\{\sigma^{\mathrm{II}}\right\}=\left\{\begin{array}{l}
\sigma_{r}^{\mathrm{II}} \\
\sigma_{\theta}^{\mathrm{II}}
\end{array}\right\} ; \quad\left\{\epsilon_{0}^{\mathrm{T}}\right\}=\left\{\begin{array}{ll}
\alpha_{0} & T^{\mathrm{II}} \\
\alpha_{0} & T^{\mathrm{II}}
\end{array}\right\}, \\
{\left[D_{0}\right]^{-1}=\frac{1}{E_{0}}\left\{\begin{array}{rr}
1 & -\nu \\
-\nu & 1
\end{array}\right\},}
\end{array}
$$

$E_{0}$ and $\alpha_{0}$ are the values of $E$ and $\alpha$ at the temperature $T_{0}$. The continuity conditions at the interface are $\sigma_{r}=\sigma_{r}^{\mathrm{II}} ; \quad \sigma_{\theta}=\sigma_{\theta}^{\mathrm{II}} ; \quad\left(u=u^{\mathrm{II}}\right), \quad$ at $r=b$.

The following non-dimensional quantities are introduced in addition to those in the previous section:

$$
\begin{array}{cc}
\tilde{\sigma}_{r}=\frac{\sigma_{r}}{\alpha_{0} T_{0} E_{0}} ; \quad \bar{\sigma}_{\theta}=\frac{\sigma_{\theta}}{\alpha_{0} E_{0} T_{0}} ; \quad \bar{\epsilon}_{r}=\frac{\epsilon_{r}}{\alpha_{0} T_{0}}, \\
\bar{\epsilon}_{\theta}=\frac{\epsilon_{\theta}}{\alpha_{0} T_{0}} . \quad \bar{E}=\frac{E}{E_{0}} ; \quad \bar{\alpha}=\frac{\alpha}{\alpha_{0}} ;
\end{array}
$$


All the equation and conditions of eqs. (17) - (27) are expressed in terms of the dimensionless quantities defined by eqs. ( 9 ) and (28).

Assuming that $\bar{\sigma}_{r}(\bar{r}=1, \bar{t})$ is known, the solution for region II is given by the following formulae [24]:

$$
\begin{aligned}
\bar{\sigma}_{r}^{\mathrm{II}} & =-\frac{1}{\bar{r}^{2}} \int_{1}^{\bar{r}}\left(\bar{r} \bar{T}^{\mathrm{II}}\right) \mathrm{d} \bar{r}+\frac{1}{\bar{r}^{2}} \frac{\bar{r}^{2}-1}{\bar{d}^{2}-1} \int_{1}^{\bar{d}}\left(\bar{r} \bar{T}^{\mathrm{II}}\right) \mathrm{d} r \\
& +\frac{1}{\bar{r}^{2}} \frac{\bar{d}^{2}-\bar{r}^{2}}{\bar{d}^{2}-1} \bar{\sigma}_{r}(\bar{r}=1, \bar{t}) \\
\bar{\sigma}_{\theta}^{\mathrm{II}} & =\frac{1}{\bar{r}^{2}} \int_{1}^{\bar{r}}\left(\bar{r} \bar{T}^{\mathrm{Il}}\right) \mathrm{d} \bar{r}-\bar{T}^{\mathrm{II}}+\frac{1}{\bar{r}^{2}} \frac{\bar{r}^{2}+1}{\bar{d}^{2}-1} \int_{1}^{\bar{d}}\left(\bar{r} \bar{T}^{\mathrm{II}}\right) \mathrm{d} \bar{r} \\
& +\frac{1}{\bar{r}^{2}} \frac{\bar{r}^{2}+\bar{d}^{2}}{\bar{d}^{2}-1} \bar{\sigma}_{r}(\bar{r}=1, \bar{t}) .
\end{aligned}
$$

The radial stress component, eq. (29), automatically satisfies the first condition of eq. (27). The second condition of eq. (27) gives, with the help of eq. (30), the following formula:

$$
\begin{aligned}
& \bar{\sigma}_{\theta}(\bar{r}=1, \bar{t})=\frac{2}{\bar{d}^{2}-1} \int_{1}^{\bar{d}}\left(\bar{r} \bar{T}^{\mathrm{II}}\right) \mathrm{d} \bar{r} \\
& -\frac{\bar{d}^{2}+1}{\bar{d}^{2}-1} \bar{\sigma}_{r}(\bar{r}=1, \bar{t})-\bar{T}(\bar{r}=1, \bar{t})
\end{aligned}
$$

which is a boundary condition at $\bar{r}=1$ for region I. Therefore, adding the condition eq. (31) to eqs. (17) - (21) we have the complete set of equations and conditions to solve the stress analysis problem for region $\mathrm{I}$.

To make the computation convenient, the differential equations and conditions for region I are transformed to those in the incremental form. Let $\Delta \bar{t}$ be a time increment between the time $\bar{t}_{j}$ and $\bar{t}_{j+1}$. Then the quantities at $\bar{t}=\bar{t}_{j+1}$ can be expressed by their increments and by their previous values as follows:

$$
\begin{array}{ll}
\left.\bar{\sigma}_{r}\right|_{\bar{t}_{j+1}}=\left.\bar{\sigma}_{r}\right|_{\bar{t}_{j}}+\Delta \bar{\sigma}_{r} ; & \left.\bar{\sigma}_{\theta}\right|_{\bar{t}_{j+1}}=\left.\bar{\sigma}_{\theta}\right|_{\bar{t}_{j}}+\Delta \bar{\sigma}_{\theta}, \\
\left.\bar{\epsilon}_{r}\right|_{\bar{t}_{j+1}}=\left.\bar{\epsilon}_{r}\right|_{\bar{t}_{j}}+\Delta \bar{\epsilon}_{r} ; & \left.\bar{\epsilon}_{\theta}\right|_{\bar{t}_{j+1}}=\left.\bar{\epsilon}_{\theta}\right|_{\bar{t}_{j}}+\Delta \bar{\epsilon}_{\theta}, \\
\left.\bar{\alpha}\right|_{\bar{t}_{j+1}}=\left.\bar{\alpha}\right|_{\bar{t}_{j}}+\Delta \tilde{\alpha} ; & \left.\bar{E}\right|_{\bar{t}_{j+1}}=\left.\bar{E}\right|_{\bar{t}_{j}}+\Delta \bar{E} ; \\
\left.\bar{T}\right|_{\bar{t}_{j+1}}=\left.\bar{T}\right|_{\bar{t}_{j}}+\Delta \bar{T} . &
\end{array}
$$

We can rewrite the equilibrium and compatibility equations in the incremental form:

$$
\begin{aligned}
& \frac{\partial\left(\Delta \bar{\sigma}_{r}\right)}{\partial \bar{r}}+\frac{\Delta \bar{\sigma}_{r}-\Delta \bar{\sigma}_{\theta}}{\bar{r}}=0, \\
& \frac{\partial\left(\Delta \bar{\epsilon}_{\theta}\right)}{\partial \bar{r}}+\frac{\Delta \bar{\epsilon}_{\theta}-\Delta \bar{\epsilon}_{r}}{\bar{r}}=0 .
\end{aligned}
$$

Substituting eq. (32) into eq. (21), we obtain

$$
\begin{aligned}
& \left\{\begin{array}{l}
\bar{\epsilon}_{r}+\Delta \bar{\epsilon}_{r} \\
\bar{\epsilon}_{\theta}+\Delta \bar{\epsilon}_{\theta}
\end{array}\right\}=\frac{1}{\bar{E}+\Delta \bar{E}}\left[\begin{array}{rr}
1 & -\nu \\
-\nu & 1
\end{array}\right]\left\{\begin{array}{l}
\bar{\sigma}_{r}+\Delta \bar{\sigma}_{r} \\
\bar{\sigma}_{\theta}+\Delta \bar{\sigma}_{\theta}
\end{array}\right\} \\
& +\left\{\begin{array}{l}
(\bar{\alpha}+\Delta \bar{\alpha})(\bar{T}+\Delta \bar{T}) \\
(\bar{\alpha}+\Delta \bar{\alpha})(\bar{T}+\Delta \bar{T})
\end{array}\right\} .
\end{aligned}
$$

Expanding the incremental quantities in Taylor series and taking their first order terms we get the stressstrain relation in the incremental form:

$$
\{\Delta \bar{\epsilon}\}=[\bar{D}]^{-1}\{\Delta \bar{\sigma}\}+[\Delta \bar{D}]^{-1}\{\bar{\sigma}\}+\left\{\Delta \bar{\epsilon}^{\mathrm{T}}\right\},
$$

where

$$
\begin{aligned}
& \{\Delta \bar{\epsilon}\}=\left\{\begin{array}{l}
\Delta \bar{\epsilon}_{r} \\
\Delta \bar{\epsilon}_{\theta}
\end{array}\right\} ; \quad\{\Delta \bar{\sigma}\}=\left\{\begin{array}{l}
\Delta \bar{\sigma}_{r} \\
\Delta \bar{\sigma}_{\theta}
\end{array}\right\} ; \\
& \left\{\Delta \bar{\epsilon}^{\mathrm{T}}\right\}=\left\{\begin{array}{l}
\bar{\alpha} \Delta \bar{T}+\bar{T} \Delta \bar{\alpha} \\
\bar{\alpha} \Delta \bar{T}+\bar{T} \Delta \bar{\alpha}
\end{array}\right), \quad[\bar{D}]^{-1}=\frac{1}{\bar{E}}\left[\begin{array}{rr}
1 & -\nu \\
-\nu & 1
\end{array}\right] ; \\
& {[\Delta \bar{D}]^{-1}=-\frac{\Delta \bar{E} / \bar{E}}{\bar{E}}\left[\begin{array}{rr}
1 & -\nu \\
-\nu & 1
\end{array}\right] .}
\end{aligned}
$$

It is noted that the second term in eq. (35) represents the effect of the temperature-dependency of Young's modulus. Substituting the stress-strain relation eq. (35) into the compatibility equation (34) and combining with the equilibrium equation (33), the differential equation which governs region $I$ is obtained in vector form:

$[S] \frac{\partial}{\partial \bar{r}}\{\Delta \bar{\sigma}\}+[W]\{\Delta \bar{\sigma}\}+\{Z\}=0$,

where the matrices $[S]$ and $[W]$ are given by

$$
\begin{aligned}
& {[S]=\frac{1}{\bar{E}}\left[\begin{array}{cc}
\bar{E} & 0 \\
-\nu & 1
\end{array}\right],} \\
& {[W]=\frac{1}{\bar{r}}\left[\begin{array}{ll}
1 & -1 \\
-\left\{\frac{1+\nu}{\bar{E}}+\nu \bar{r} \frac{\partial}{\partial \bar{r}}\left(\frac{1}{\bar{E}}\right)\right\} & \frac{1+\nu}{\bar{E}}+\bar{r} \frac{\partial}{\partial \bar{r}}\left(\frac{1}{\bar{E}}\right)
\end{array}\right],}
\end{aligned}
$$


and the vector $\{Z\}$ is in the form

$$
\{Z\}=\left\{\begin{array}{l}
0 \\
Z_{2}
\end{array}\right\},
$$

where

$$
\begin{aligned}
Z_{2} & =\frac{\Delta \bar{E} / \bar{E}}{\bar{r}} \frac{1+\nu}{\bar{E}}\left(\bar{\sigma}_{\theta}-\bar{\sigma}_{r}\right) \\
& +\frac{\partial}{\partial \bar{r}}\left[\frac{\Delta \bar{E} / \bar{E}}{\bar{E}}\left(\bar{\sigma}_{\theta}-\nu \bar{\sigma}_{r}\right)+(\bar{\alpha} \Delta \bar{T}+\bar{T} \Delta \bar{\alpha})\right] .
\end{aligned}
$$

We use the same mesh system as that for the thermal analysis. The differential equation (36) for the stress field is converted into the finite difference form:

$$
\begin{aligned}
& {[S] \frac{\{\Delta \bar{\sigma}\}\left|\bar{r}_{i}-\{\Delta \bar{\sigma}\}\right| \bar{r}_{i-1}}{\bar{r}_{i}-\bar{r}_{i-1}}+[W] \frac{\{\Delta \bar{\sigma}\}\left|\bar{r}_{i}+\{\Delta \bar{\sigma}\}\right| \bar{r}_{i-1}}{2}} \\
& \quad+\{Z\}=0 .
\end{aligned}
$$

Eq. (37) is expressed in the global form

$[\tilde{A}]\{\Delta \bar{\sigma}\}=\{\tilde{B}\}$,

where $\{\Delta \bar{\sigma}\}$ consists of $\{\Delta \bar{\sigma}\}$ at the discre te points.

\section{Numerical representation of properties}

Fig. 2 shows the thermal and mechanical properties of 1045 steel used in the computations. All the properties except the Young's modulus are taken from ref. [25]. The temperature-dependency of Young's modulus is taken from ref. [26]. Theoretically Young's modulus becomes zero in the melting phase but, for computational convenience, we assume a small value for $\bar{E}$ instead of zero.

The thermal expansion coefficient generally depends on temperature and on the rate of change of temperature [27]; however, the latter dependency is neglected in this paper.

Using the Gauss elimination method on eq. (16), the temperature distributions are calculated at each time step. Time begins with the process where the inner boundary is heated above the melting temperature and followed by the cooling process in which the inner boundary is cooled down from the saturated temperature at the end of the heating process to the room temperature.

When carrying out the solution numerically the

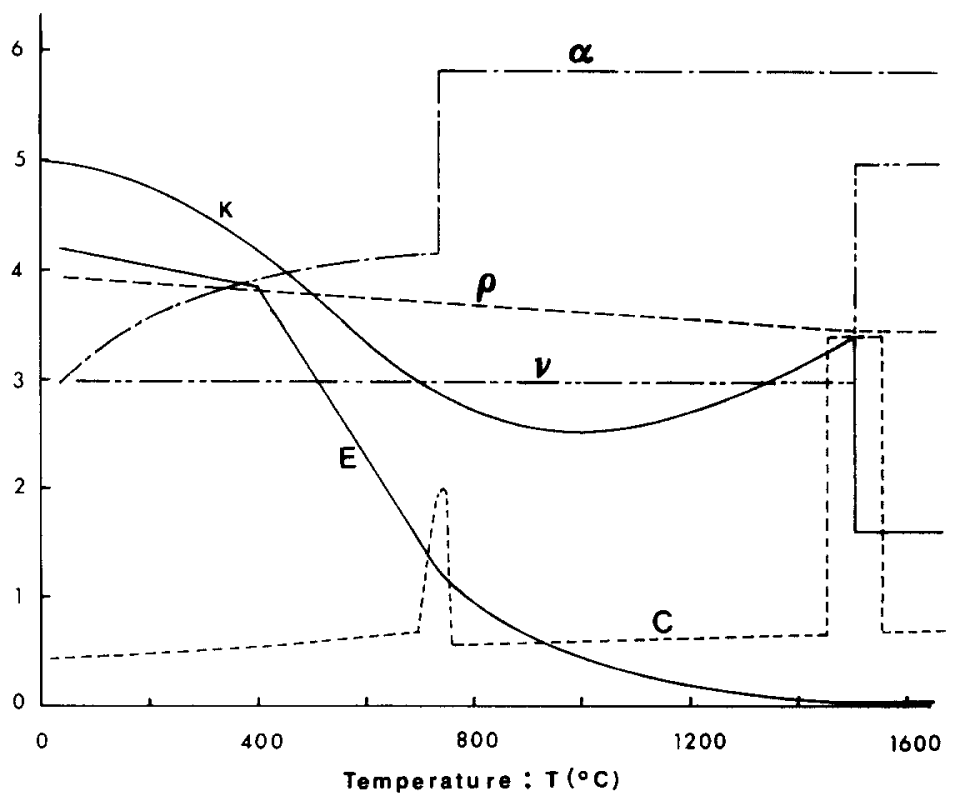

Fig. 2. Thermal and mechanical properties with respect to temperature for a carbon steel. Density: $\rho \times 2$ (g/cm ${ }^{3}$ ), Poisson's ratio: $\nu \times 10^{-1}$, Thermal expansion coefficient: $\alpha \times\left(4 \times 10^{-6}\right)\left(1 /{ }^{\circ} \mathrm{C}\right)$, Young's modulus: $E \times 10^{6}\left(\mathrm{~kg} / \mathrm{cm}^{2}\right)$, Thermal conductivity: $K \times 10^{-3}\left(\mathrm{~W} / \mathrm{cm}^{\circ} \mathrm{C}\right)$, Specific heat: $C\left(\mathrm{~J} / \mathrm{g}^{\circ} \mathrm{C}\right)$. 
liquid-solid interface may not coincide with a mesh point. Some interpolation techniques were used [28] for accuracy. We assume the coincidence of the interface and a mesh point in this analysis by using a fine mesh system.

\section{Numerical results and discussions}

A thin plate of $\bar{a}=0.1$ and $\bar{d}=3.0$, having the properties of the carbon steel 1045 as shown in fig. 2 , is considered. We use the initial temperature $T_{0}=30^{\circ} \mathrm{C}$, the Biot number $\mathrm{Bi}=12.5$ and $T_{\mathrm{s}}=2000^{\circ} \mathrm{C}$.

Fig. 3 shows the change of the tangential stress and temperature at the inner boundary $\bar{r}=\bar{a}$ as functions of time during the heating process. It is predicted that the rate of increase of $\bar{\sigma}_{\theta}$ at the initial stage greatly depends on the Biot number. The material softening recognizably appears on $\bar{\sigma}_{\theta}$ from $\bar{t}=10^{-4}$. When the inner boundary changes its crystal structure from the body-centered cubic to the face-centered cubic struc- ture $\bar{t}=10^{-3}, \bar{\sigma}_{\theta}$ shows a concave trace with respect to time. After the phase transformation of the crystal structure, the temperature continues to increase and the influence of material softening on the stress is more pronounced. $\bar{\sigma}_{\theta}$ decreases rapidly as the temperature approaches the melting state of the material. When $\bar{T}$ rises beyond the melting temperature, $\bar{\sigma}_{\theta}$ reaches a small value. (It should be zero theoretically, but in this analysis we assumed a finite, small value of the Young's modulus in the melting state in order to avoid the computational difficulty.)

During the cooling process $\bar{\sigma}_{\theta}$ and $\bar{T}$ on the inner boundary with respect to time are shown in fig. 4 , where $\bar{t}=0$ is taken as the start of cooling at the inner boundary. The region near the inner boundary is subjected to the solidification process at an early stage and then to the phase transformation of the crystal structures. $\bar{T}$ drops quickly before the crystal structure changes, i.e., $\bar{t}=10^{-2}, \bar{\sigma}_{\theta}$ reaches the maximum tensile value. After the crystal structure changes, the location of the maximum temperature drop is

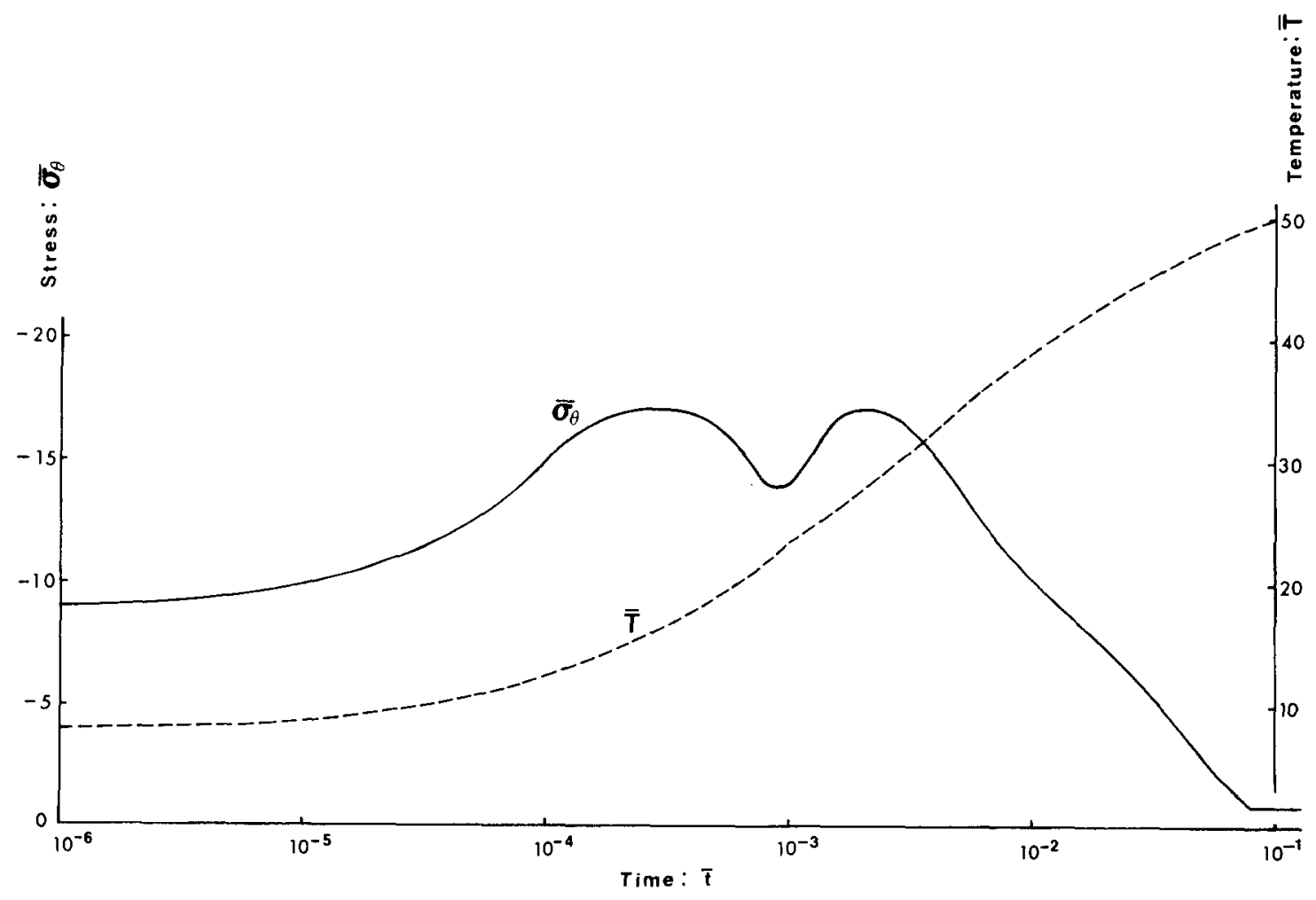

Fig. 3. Transient temperature and tangential stress at the inner boundary during the heating process. 


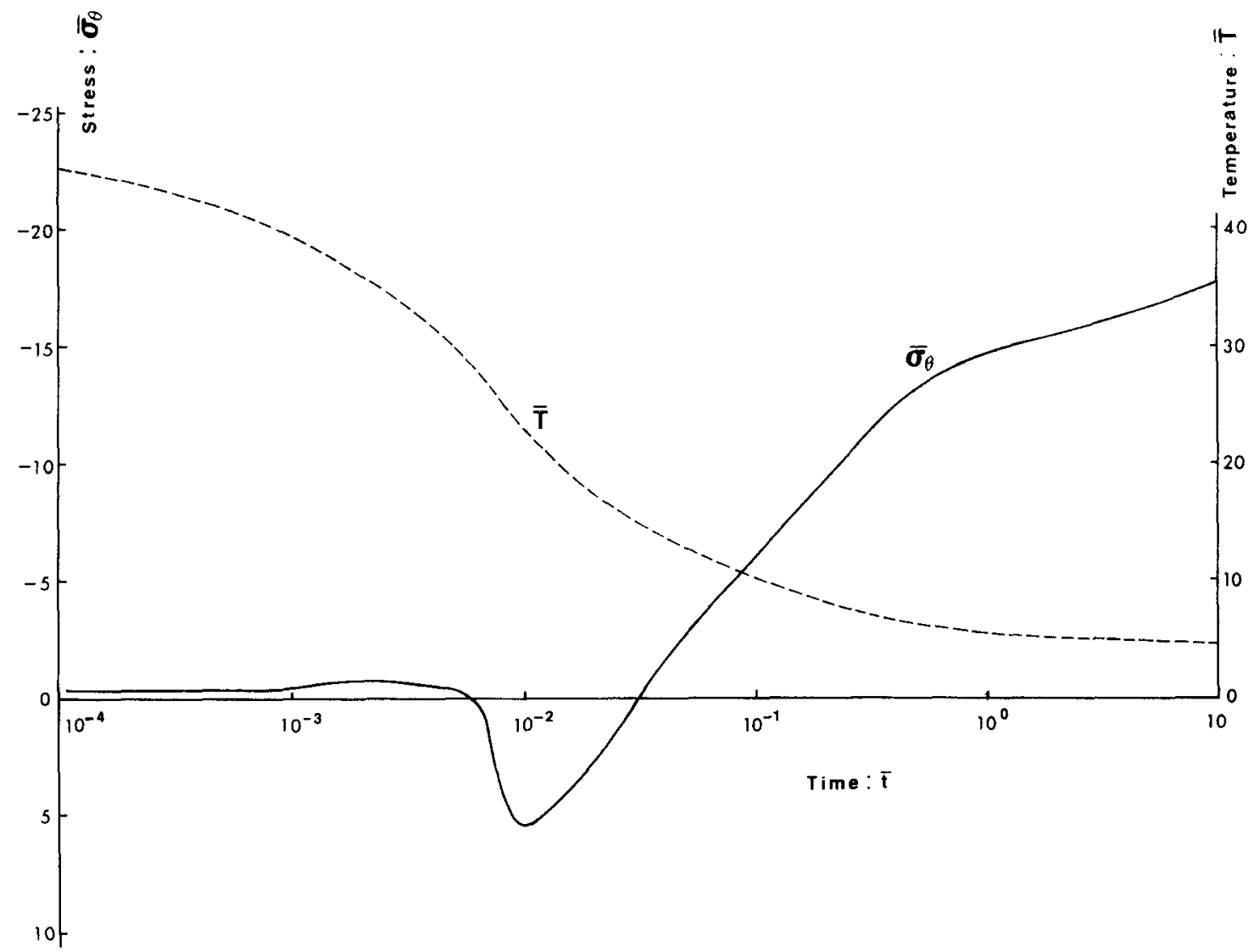

Fig. 4. Transient temperature and tangential stress at the inner boundary during the cooling process.

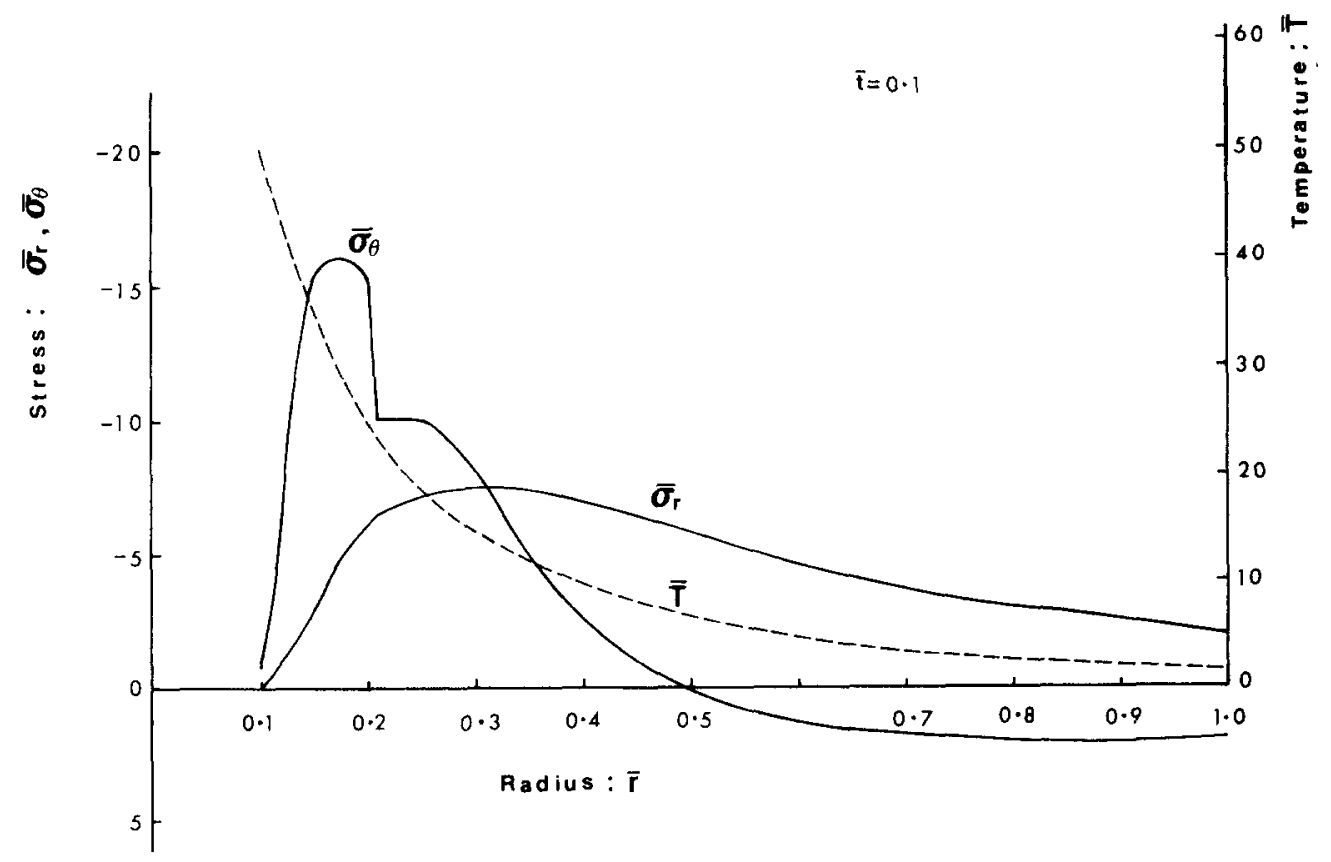

Fig. 5. Temperature, tangenital and radial stress distributions at $t=0.1$ during the heating process. 


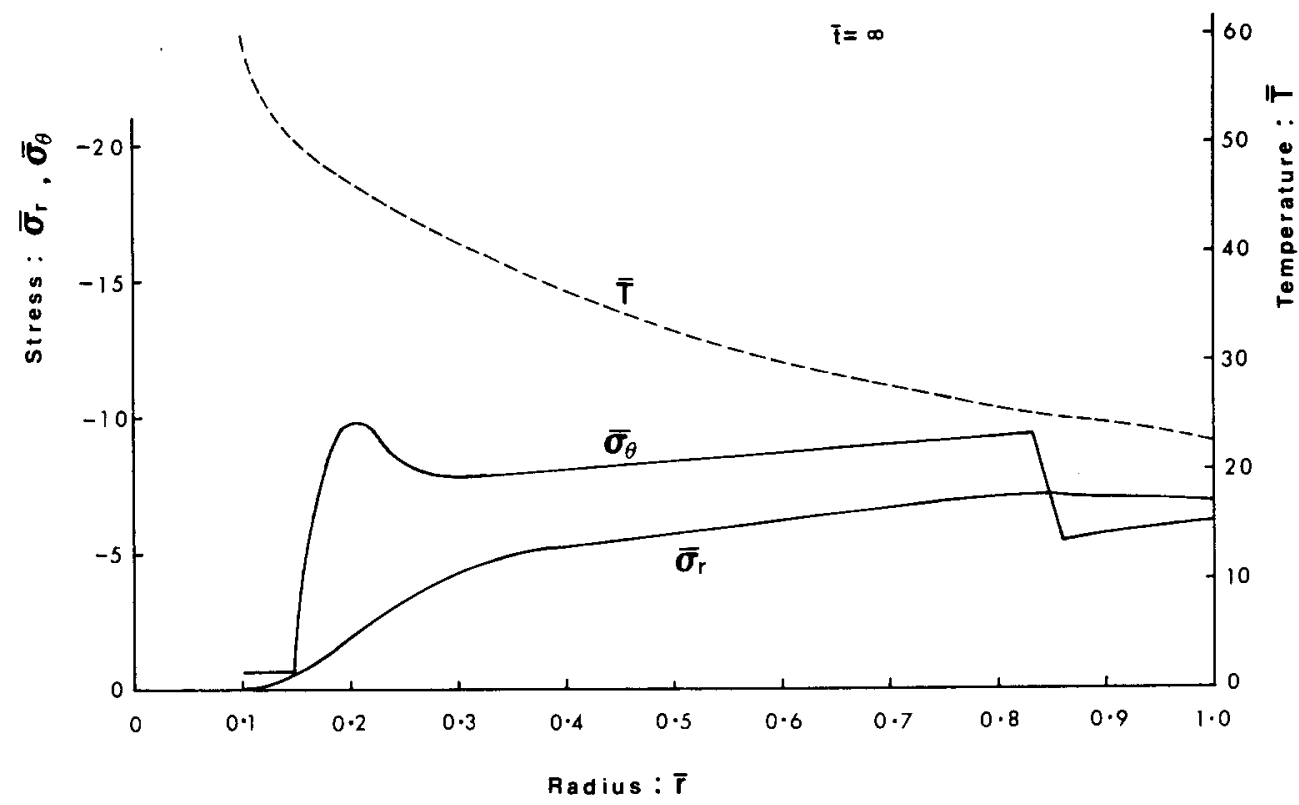

Fig. 6. Temperature, tangenital and radial stress distributions at $t=\infty$ during the heating process.

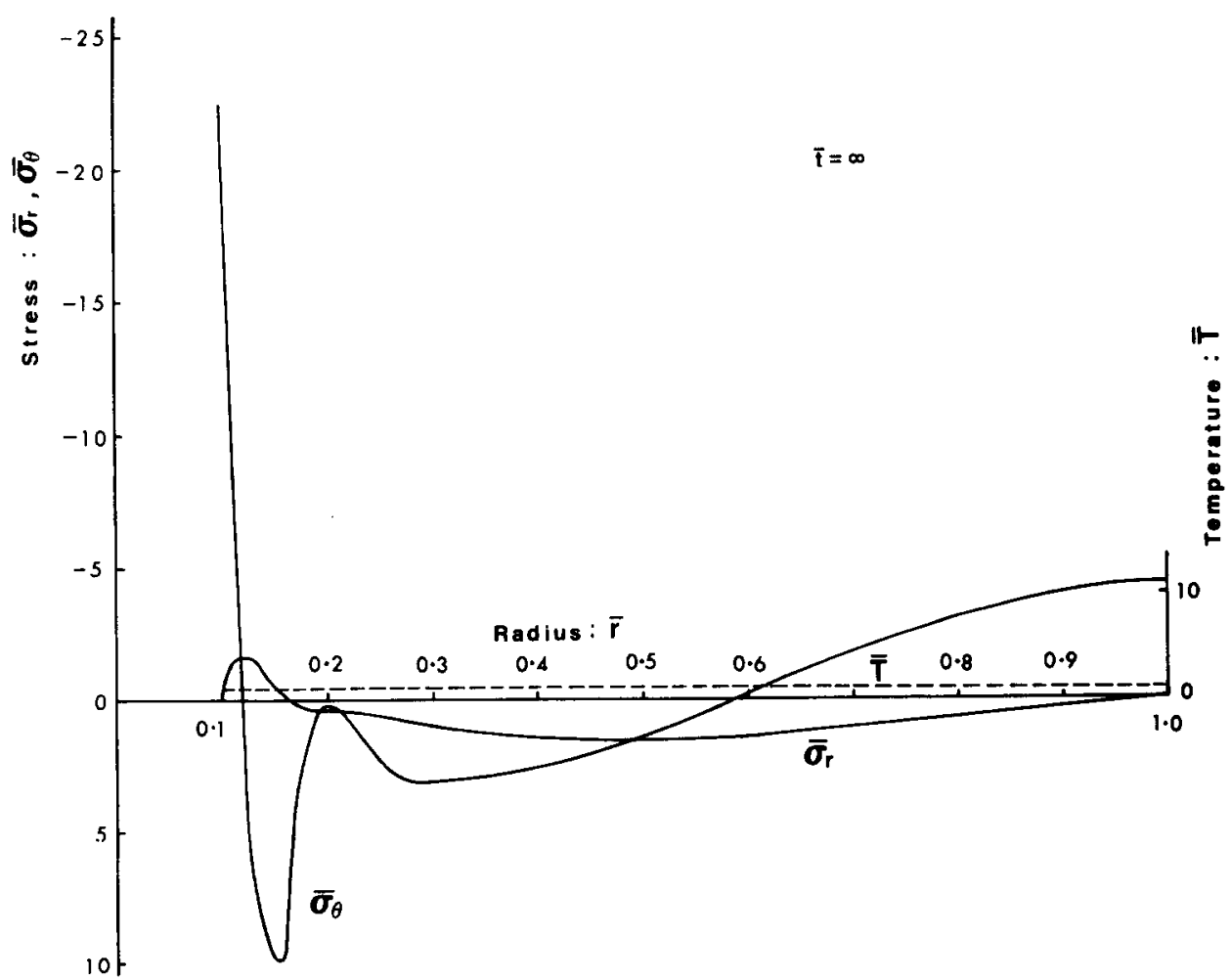

Fig. 7. Residual and tangential stress distributions. 
shifted from a point near the inner boundary to an interior point and $\bar{\sigma}_{\theta}$ becomes compressive. When the inner boundary approaches the room temperature, $\bar{\sigma}_{\theta}$ becomes more compressive.

It can be seen in both fig. 3 and fig. 4 that $\bar{\sigma}_{\theta}$ changes remarkably around the crystal structural changes.

Fig. 5 shows the tangential and radial stress distributions at $\bar{t}=0.1$ where the dotted line indicates the temperature distribution. Since the phase transformation of the crystal structures occurs at $\bar{T}=25$, it takes place at $\bar{r}=0.2$ where the tangential stress drops suddenly. $\bar{\sigma}_{\theta}$ decreases in the region near the inner boundary due to the material softening. It can be seen that the radial stress is not as sensitive as the tangential stress to the phase transformation of the crystal structures.

The steady state of stress and temperature distributions in the heating process, that is $\bar{t}=\infty$, are shown in fig. 6 . The melting front reaches to $\bar{r}=0.15$ and the crystal structural changes appear around $\bar{r}=0.85$.

Fig. 7 shows the distribution of both the tangential and radial components of the residual stress. $\bar{\sigma}_{\theta}$ changes steeply in the region $0.1 \leqslant \bar{r} \leqslant 0.2$ where the melting phase took place at the final stage of the heating process. It is interesting to point out that the residual distribution of $\bar{\sigma}_{\theta}$ in the region having experienced the melting phase once is similar to that produced by a thermal shock [28].

\section{Conclusions}

The effects of the phase changes on the stresses and temperature in an infinite plate were studied using the non-linear elasticity and non-linear diffusion theory. The numerical results concerning the stresses led to the following conclusions.

(1) Around the phase transformation of the crystal structures the tangential stress changes rapidly in time space and geometrical space.

(2) After one cycle of thermal loading the residual tangential and radial stresses were obtained. The tangential component of the residual stress changes steeply in the region where the melting phase occurred at the end stage of the heating process.

The thermal and stress analyses provide information on metallurgical and mechanical states of the material after one cycle of over-heating in terms of melting phase penetration, local heating and cooling history, and residual stresses. This information is essential to the design of nuclear reactors with an accident proof safety margin.

\section{References}

[1] K. Masubuchi, Weld. Res. Counc., no. 149 (1970).

[2] R.B. Knapp and N.E. Todreas, Nucl. Eng. Des. 35 (1975) 69.

[3] K. Masubuchi, NASA, TMX-61300 (1968).

[4] B.A. Boley and A. Lahoud, Nucl. Eng. Des. 32 (1975) 1.

[5] H.S. Carslaw and J.C. Jaeger, Conduction of Heat in Solids (Oxford University Press, 1959).

[6] B.A. Boley, Nucl. Eng. Des. 18 (1972) 377.

[7] J.R. Ockendon and W.R. Hodgkins, Moving Boundary Problems in Heat Flows and Diffusion (Oxford University Press, 1975).

[8] P. Perzyna and A. Sawczuk, Nucl. Eng. Des. 24 (1973) 1.

[9] J.H. Weiner and J.V. Huddleston, J. Appl. Mech. 26 (1959) 31.

[10] H.G. Landau and J.H. Weiner, J. Appl. Mech. 31 (1964) 148.

[11] G.V. Plyatsko and B.G. Schelestovski, Sov. Mater. Sci. 7 (1971) 51 .

[12] V.I. Goisa, Appl. Mech. Math. 38 (1974) 842.

[13] Y. Iwamura and E.F. Rybicki, J. Eng. Ind. 95 (1973) 163.

[14] T. Inoue and K. Tanaka, Int. J. Mech. Eng. 17 (1975) 361.

[15] E. Friedman and B.A. Boley, J. Space Rockets 7 (1970) 324.

[16] R.I. Pedroso and G.A. Domoto, J. Eng. Mater. Technol. 95 (1973) 227.

[17] I.S. Habib, J. Appl. Mech. 40 (1973) 307.

[18] M. Levitsky and B.W. Shaffer, J. Appl. Mech. 41 (1974) 647.

[19] O. Richmond and R.H. Tien, J. Mech. Phys. Solids 19 (1971) 273.

[20] J.H. Weiner and B.A. Boley, J. Mech. Phys. Solids 11 (1963) 145

[21] E. Friedman, J. Pressure Vessel Technol. 97 (1975) 206.

[22] H.D. Hibbit and P.V. Marcal, Comput. Struct. 3 (1973) 1145.

[23] M.N. Ozisik, Boundary Value Problems of Heat Conduction (International Textbook Co., 1968).

[24] B.A. Boley and J.H. Weiner, Theory of Thermal Stresses (John Wiley, 1960).

[25] Y.S. Toulouskian, Thermophysical Properties of High Temperature Solid Materials, vol. 3 (MacMillan, 1967).

[26] C.L. Clark, High Temperature Alloys (Sir Issac Pitman and Sons, 1953).

[27] T. Inoue and K. Tanaka, Kyoto Daigaku Kogakubu Memoir of Faculty 35 (1973) 1.

[28] J. Crank, in: Moving Boundary Value Problems in Heat Flow and Diffusion, J.R. Ockendon and W.R. Hodgkins, eds. (Oxford University Press, 1975) pp. 192-207. 